\title{
ON CERTAIN CLASSES OF CLOSE-TO-CONVEX FUNCTIONS
}

KHALIDA INAYAT NOOR

\author{
Mathematics Department, College of Science, \\ P.O. Box 2455, King Saud University, \\ Riyadh 11451, Saudi Arabia
}

(Received May 30, 1991 and in revised form September 27, 1991)

\begin{abstract}
A function $f$, analytic in the unit disk $E$ and given by $f(z)=z+\sum_{k=2}^{\infty} a_{n} z^{k}$ is said to be in the family $K_{n}$ if and only if $D^{n} f$ is close-to-convex, where $D^{n} f=\frac{z}{(1-z)^{n+1}} * f, n \in N_{0}=\{0,1,2, \ldots\}$ and * denotes the Hadamard product or convolution. The classes $K_{n}$ are investigated and some properties are given. It is shown that $K_{n+1} \subseteq K_{n}$ and $K_{n}$ consists entirely of univalent functions. Some closure properties of integral operators defined on $K_{n}$ are given.
\end{abstract}

KEY WORDS AND PHRASES. Univalent, close-to-convex, starlike, convolution, integral operators.

1991 AMS SUBJECT CLASSIFICATION CODES. 30C45, 30A32.

\section{INTRODUCTION.}

Let $A$ denote the class of functions $f: f(z)=z+\sum_{k=2}^{\infty} a_{k} z^{k}$ analytic in the unit disk $E=\{z:|z|<1\}$. The Hadamard product or convolution of two functions $f, g \in A$ is denoted by $f * g$. For $n \in N_{0}=\{0,1,2,3, \ldots\}$, let $D^{n} f=\left(\frac{z}{(1-z)^{n+1}} * f\right.$, so that

$$
D^{n} f=z\left(z^{n-1} f\right)^{(n)} / n !
$$

Let $S \subset A$ be the class of univalent functions and for $0 \leq \beta<1$, let $C(\beta)$ and $S^{*}(\beta)$ denote the subclasses of $S$ consisting of convex functions of order $\beta$ and starlike functions of order $\beta$ respectively. The classes $C$ and $S^{*}$ of convex and starlike functions, respectively, are identified by $C(0) \equiv C$ and $S^{*}(0)=S^{*}$.

A function $f \in S$ belongs to the class $K(\alpha, \beta)$ of close-to-convex of order $\alpha$ and type $\beta$ if and only if for some $g \in S^{*}(\beta)$ and $0 \leq \alpha<1$,

$$
\operatorname{Re} \frac{z f^{\prime}(z)}{g(z)}>\alpha, z \in E
$$

It is clear that $K(0,0) \equiv K$, the class of close-to-convex univalent functions [1].

DEFINITION 1.1. For $n \in N_{0}$, a function $f \in A$ is said to belong to the classes $R_{n}$, if and only if for $z \in E$,

$$
\operatorname{Re} \frac{z\left(D^{n} f(z)\right)^{\prime}}{D^{n} f(z)}>0
$$

Thus $R_{0} \equiv S^{*}$ and $R_{1} \equiv C$. In [2], Ahuja discussed these classes and showed that $R_{n+1} \subset R_{n}$ for 
each $n \in N_{0}$. This implies that functions in $R_{n}$ are starlike and hence univalent.

We now extend the classes $R_{n}$, as follows:

DEFINITION 1.2. Let $f \in A$. Then $f \in K_{n}$ if and only if there exists $g \in R_{n}$ such that for $z \in E$,

$$
\operatorname{Re} \frac{z\left(D^{n} f(z)\right)^{\circ}}{D^{n} g(z)}>0
$$

We note that $K_{0} \equiv K$ and $K_{1} \equiv C^{*}$, the class of quasi-convex functions introduced in [3].

In order to develop some results for $K_{n}$, we shall need the following:

LEMMA 1.1 [4]. Let $w$ be analytic in $E$. If $|w|$ assumes its maximum value on the circle $|z|=r$ at a point $z_{0}$, then

$$
z_{0} \omega^{\prime}\left(z_{0}\right)=k \omega\left(z_{0}\right)
$$

where $k \geq 1$.

LEMMA 1.2 [5]. Let $u=u_{1}+i u_{2}$ and $v=v_{1}+i v_{2}$ and $\psi(u, v)$ be a complex-valued function satisfying the conditions:

(i) $\psi(u, v)$ is continuous in a domain $D \subset C^{2}$,

(ii) $(1,0) \in D$ and $\psi(1,0)>0$,

(iii) $\operatorname{Re}\left(i u_{2}, v_{1}\right) \leq 0$ whenever $\left(i u_{2}, v_{1}\right) \in D$ and $v_{1} \leq-\frac{1}{2}\left(1+u_{2}^{2}\right)$.

If $h(z)=1+\sum_{k=2}^{\infty} c_{k} z^{k}$ is a function analytic in $E$, such that $\left(h(z), z h^{\prime}(z)\right) \in D$ and $R e \psi\left(h(z), z h^{\prime}(z)\right)>0$ for $z \in E$, then $\operatorname{Re} h(z)>0$ in $E$.

LEMMA 1.3. [6]. Let $\phi$ be convex and $g$ be starlike in $E$. Then, for $F$ analytic in $E$ with $F(0)=1, \frac{\phi^{*} F g}{\phi^{*} g}$ is contained in the convex hull of $F(E)$.

2. PROPERTIES OF THE FAMILY $K_{n}$.

We first prove that all functions in $K_{n}$ are close-to-convex and hence univalent.

THEOREM 2.1. $K_{n+1} \subset K_{n}$, for each $n \in N_{0}$.

PROOF. Let $f \in K_{n+1}$. Then for $z \in E$

$$
R e \frac{z\left(D^{n+1} f(z)\right)^{\prime}}{D^{n+1} g(z)}>0, \text { for some } g \in R_{n+1} \text {. }
$$

Define $\omega(z)$ in $E$ such that

$$
\frac{z\left(D^{n} f(z)\right)^{\prime}}{\left.D^{n} g(z)\right)}=\frac{1-\omega(z)}{1+\omega(z)}
$$

where $\omega(0)=0$ and $\omega(z) \neq .-1$. We show that $|\omega(z)|<1$.

From (2.1) we have

$$
z\left(D^{n} f(z)\right)^{\prime}=D^{n} g(z) \cdot \frac{1-\omega(z)}{1+\omega(z)^{\prime}}
$$

So, from (2.2) and the identity

we obtain

$$
z\left(D^{n} f(z)\right)^{\circ}=(n+1) D^{n+1} f(z)-n D^{n} f(z),
$$

$$
z\left(D^{n+1} f(z)\right)^{\prime}=\frac{1}{n+1}\left[z\left(D^{n} g(z)\right)^{\prime} \frac{1-\omega(z)}{1+\omega(z)}+D^{n} g(z)\left\{\frac{-2 z \omega^{\prime}(z)}{(1+\omega(z))^{2}}+n \frac{1-\omega(z)}{1+\omega(z)}\right\}\right]
$$

Now apply (2.3) for the function $g$, and use (2.4) to obtain

$$
\frac{z\left(D^{n+1} f(z)\right)^{\prime}}{D^{n+1} g(z)}=\frac{1-\omega(z)}{1+\omega(z)}+\frac{1}{n+1} \frac{D^{n} g(z)}{D^{n+1} g(z)} \cdot\left[\frac{-2 z \omega^{\prime}(z)}{(1+\omega(z))^{2}}\right]
$$


Since $R_{n+1} \subset R_{n}$, this implies that $g \in R_{n}$ and hence there exists an analytic function $\omega_{1}(z)$ with $\omega_{1}(0)=0$ and $\left|\omega_{1}(z)\right|<1$ such that

$$
\frac{D^{n+1} g(z)}{D^{n} g(z)}=\frac{1-\omega(z)}{1+\omega(z)}
$$

Thus using (2.6) in (2.5) we have

$$
\frac{z\left(D^{n+1} f(z)\right)^{\circ}}{D^{n+1} g(z)}=\frac{1-\omega(z)}{1+\omega(z)}+\frac{1}{n+1}\left(\frac{1+\omega_{1}(z)}{1-\omega_{1}(z)}\right)\left(\frac{-2 z \omega^{\prime}(z)}{(1+\omega(z))^{2}}\right)
$$

Suppose now that for $z \in E$

$$
|z| \leq\left|z_{0}\right| \max |\omega(z)|=\left|\omega\left(z_{0}\right)\right|=1,\left(\omega\left(z_{0}\right) \neq-1\right) .
$$

Then it follows, from Lemma 1.1, that

$$
z_{0} \omega^{\prime}\left(z_{0}\right)=k \omega\left(z_{0}\right)
$$

where $k \geq 1$.

Setting $\omega\left(z_{0}\right)=e^{i \Theta}$ and $\omega_{1}\left(z_{0}\right)=r e^{i \phi}$ in (2.7) gives

$$
\begin{aligned}
\operatorname{Re}\left\{\frac{z_{0}\left(D^{n+1} f\left(z_{0}\right)\right)^{\prime}}{D^{n+1} g\left(z_{0}\right)}\right\} & =\operatorname{Re}\left[\left(\frac{1}{n+1}\right) \frac{-2 k\left(e^{i \Theta}+e^{-i \Theta}+2\right)\left(1+r^{2}+2 r \cos \phi\right)}{\left|1+r e^{i \phi}\right|^{2}\left|\left(1+e^{i \Theta}\right)^{2}\right|^{2}}\right] \\
& =\frac{-4 k}{n+1}\left[\frac{(\cos \Theta+1)\left(1+r^{2}+2 r \cos \phi\right)}{\left|1+r e^{i \phi}\right|^{2}\left|\left(1+e^{i \Theta}\right)^{2}\right|^{2}}\right]
\end{aligned}
$$

Hence, if $\phi=\frac{\pi}{2}$,

$$
\operatorname{Re} \frac{z_{0}\left(D^{n+1} f\left(z_{0}\right)\right)^{\prime}}{D^{n+1} g\left(z_{0}\right)}<0,
$$

where $g \in R_{n+1}$ and $k \geq 1$. This contradicts our hypothesis that $f \in K_{n+1}$. Thus $|\omega(z)|<1$ and so $f \in K_{n}$.

From Theorem 2.1, we note that $f \in K_{n}$ implies that $f \in K$ and so $f$ is univalent in $E$. Also, since $K_{n} \subset K_{1} \equiv C^{*}$ it follows that $f$ is quasi-convex.

REMARK 2.1. Let $f \in K_{n}$ and be given by $f(z)=z+\sum_{k=2}^{\infty} a_{k} z^{k}$. Then

$$
\begin{aligned}
D^{n} f(z) & =\frac{z}{(1-z)^{n+1}} * f(z), \\
& =\left[z+\sum_{k=2}^{\infty} \frac{(k+n-1) !}{n !(k-1) !} z^{k}\right] *\left[z+\sum_{k=2}^{\infty} a_{k} z^{k}\right] \\
& =z+\sum_{k=2}^{\infty} \frac{(k+n-1) !}{n !(k-1) !} a_{k} z^{k}
\end{aligned}
$$

Thus from (2.8) and Definition 1.2 it follows that

$$
f \in K_{n} \text { if and only if } D^{n} f \in K .
$$

THEOREM 2.2. Let $f \in K_{n}$ and be given by $f(z)=z+\sum_{k=2}^{\infty} a_{a} z^{k}$. Then for $k \geq 2$ and $n \geq 0$,

$$
\left|a_{k}\right| \leq \frac{(k !)(n !)}{(k+n-1) !}
$$


This result is sharp with equality for the function $f_{0}$, where

$$
D^{n} f_{0}(z)=\frac{z}{(1-z)^{2}}
$$

The proof follows immediately from (2.8), (2.9), and the well-known coefficient result for the class $K$ of close-to-convex functions.

THEOREM 2.3. (Covering theorem). Let $f \in K_{n}$. If $B$ is the boundary of the image of $E$ under $f$, then every point of $B$ is distance at least $\frac{n+1}{2(n+2)}$ from the origin.

PROOF. Let $f(z) \neq c, c \neq 0$. Then $f_{1}$ given by

$$
f_{1}(z)=\frac{c f(z)}{c-f(z)}
$$

is univalent in $E$. Write $f(z)=z+\sum_{k=2}^{\infty} a_{k} z^{k}$, then

$$
\frac{c f(z)}{c-f(z)}=z+\left(a_{2}+\frac{1}{c}\right) z^{2}+\cdots \cdots \cdots \cdots
$$

and, since $f_{1} \in S$, it follows that

$$
\left|a_{2}+\frac{1}{c}\right| \leq 2
$$

Hence,

$$
\left|\frac{1}{c}\right| \leq 2+\left|a_{2}\right|
$$

and using Theorem 2.2, we obtain

$$
|c| \geq \frac{n+1}{2(n+2)}
$$

This completes the proof.

We note that when $n=0,|c| \geq \frac{1}{4}$ and when $n=1,|c| \geq \frac{1}{2}$ (see [3] and [7]).

THEOREM 2.4. $\bigcap_{n=0}^{\infty} K_{n}=\{i d\}$,

where id is the identity function $z$.

PROOF. Let $f(z)=g(z)=z$ in (1.2), then it follows trivially that $z \in K_{n}$ for $n \geq 0$.

On the contrary, assume that $f \in \bigcap_{n=0}^{\infty} K_{n}$ with $f(z)=z+\sum_{k=2}^{\infty} a_{k} z^{k}$.

Then it follows from Theorem 2.2. that $f(z)=z$.

3. INTEGRAL OPERATORS.

Let the operator $I_{\lambda}: A \rightarrow A$ be defined by $f=I_{\lambda}(F)$, as

$$
f(z)=\frac{1}{\lambda} z^{1-\frac{1}{\lambda}} \int_{0}^{z} \xi^{\frac{1}{\lambda}-2} F(\xi) d \xi,
$$

where $0<\lambda \leq 1$.

For $\lambda=\frac{1}{2}$, Libera [8] established that the operator

$$
I(F)=\frac{2}{2} \int_{0}^{z} F(\xi) d \xi
$$

preserves convexity, starlikeness, and close-to-convexity. Bernardi [9] greatly generalized Libera's results. Many authors have studied the operators of the form (3.1), see e.g. [7]. Ahuja [2] has discussed the $\lambda=\frac{1}{\gamma+1}, \gamma$ complex and $R e \gamma \neq-1$ for the classes $R_{n}$. Here we shall consider (3.1) for $K_{n}$. 
We shall need the following [2]:

Let $I_{\lambda}: A \rightarrow A$ be defined by (3.1) with $0<\lambda \leq 1$. If $F \in R_{n}$, then $I_{\lambda}(F) \in R_{n}(\alpha)$, i.e., for $z \in E$

$$
\operatorname{Re} \frac{z\left(D^{n} f(z)\right)^{\prime}}{D^{n} f(z)}>\alpha
$$

where $0<\alpha<1$ and

$$
\alpha=\frac{1}{4 \lambda}\left[-(2-\lambda)+\sqrt{9 \lambda^{2}+4 \lambda+1}\right]
$$

We now prove:

THEOREM 3.1. Let $F \in K_{n}$ and let $f=I_{\lambda}(F)$ be defined as in (3.1) for $0<\lambda \leq 1$. Then $f \in K_{n}(\beta, \alpha)$, where $\alpha$ is given by (3.2) and $\beta(0 \leq \beta<1)$ is defined by (3.9).

PROOF. Let $G \in R_{n}$ and $I_{\lambda}(G)=g$, where $I_{\lambda}$ is defined by (3.1). So that

and

$$
D^{n} G(z)=(1-\lambda) D^{n} g(z)+\lambda z\left(D^{n} g(z)\right)^{\prime}
$$

$$
D^{n} F(z)=(1-\lambda) D^{n} f(z)+\lambda z\left(D^{n} f(z)\right)^{\prime}
$$

Set

where

$$
\frac{z\left(D^{n} f(z)\right)^{\prime}}{D^{n} g(z)}=(1-\beta) p(z)+\beta
$$

$$
p(z)=1+\sum_{n=1}^{\infty} c_{n} z^{n}
$$

We need to show that $\operatorname{Re} p(z)>0$ for $z \in E$.

From (3.3), we have

$$
\frac{z\left(D^{n} F(z)\right)^{\prime}}{D^{n} G(z)}=\frac{(1-\lambda) \frac{z\left(D^{n} f(z)\right)^{\prime}}{D^{n} g(z)}+\frac{\lambda z\left[z\left(D^{n} f(z)\right)^{\prime}\right]^{\prime}}{D^{n} g(z)}}{(1-\lambda)+\lambda z \frac{\left(D^{n} g(z)\right)^{\prime}}{D^{n} g(z)}}
$$

Since $g \in R_{n}(\alpha)$, where $\alpha$ is given by (3.2), we can write

$$
\frac{z\left(D^{n} g(z)\right)^{\prime}}{D^{n} g(z)}=(1-\alpha) p_{0}(z)+\alpha,
$$

where Re $p_{0}(z)>0, z \in E$.

Also, from (3.4) and (3.6), we obtain

$$
\begin{aligned}
\frac{z\left[z\left(D^{n} f(z)\right)^{\prime}\right]^{\prime}}{D^{n} g(z)} & =\left\{\frac{z\left(D^{n} g(z)\right)^{\prime}}{D^{n} g(z)}((1-\beta) p(z))+\beta^{z} \frac{z\left(D^{n} g(z)\right)^{\prime}}{D^{n} g(z)}+(1-\beta) z p^{\prime}(z)\right\} \\
& =\left[(1-\alpha) p_{0}(z)+\alpha\right][(1-\beta) p(z)+\beta]+(1-\beta) z p^{\prime}(z) .
\end{aligned}
$$

Using (3.4), (3.6), and (3.7) in (3.5), it follows that

$$
\frac{z\left(D^{n} F(z)\right)^{\prime}}{D^{n} G(z)}=\beta+(1-\beta) p(z)+\frac{\lambda(1-\beta) z p^{\prime}(z)}{(1-\lambda)+\lambda\left[(1-\alpha) p_{0}(z)+\alpha\right]}
$$

Next define $\psi(u, v)$ by taking $u=p(z)$ and $v=z p^{\prime}(z)$ in (3.8) by

$$
\psi(u, v)=\beta+(1-\beta) u+\frac{\lambda(1-\beta) v}{\lambda(1-\alpha) p_{0}-\lambda(1-\alpha)+1} .
$$


It is clear that $\psi(u, v)$ satisfies conditions (i) and (ii) of Lemma 1.2. To verify condition (iii), we note that

$$
\operatorname{Re} \psi\left(i u_{2}, v_{1}\right)=\beta+\frac{\lambda(1-\beta) v_{1}\left\{\lambda(1-\alpha) p_{1}-\lambda(1-\alpha)+1\right\}}{\left\{\lambda(1-\alpha) p_{1}-\lambda(1-\alpha)+1\right\}^{2}+\lambda^{2}(1-\alpha)^{2} p_{2}^{2}}
$$

where $p_{0}(z)=p_{1}+i p_{2}, p_{1}, p_{2}$ being functions of $x$ and $y$ and Re $p_{0}=p_{1}>0$.

By putting $v_{1} \leq-\frac{1}{2}\left(1+u_{2}^{2}\right)$, we obtain

$$
\operatorname{Re} \psi\left(i u_{2}, v_{1}\right) \leq \beta-\frac{\lambda(1-\beta)\left(1+u_{2}^{2}\right)\left[\lambda(1-\alpha) p_{1}-\lambda(1-\alpha)+1\right]}{2\left[\left\{\lambda(1-\alpha) p_{1}-\lambda(1-\alpha)+1\right\}^{2}+\lambda^{2}(1-\alpha)^{2} p_{2}^{2}\right]}=\frac{A+B u_{2}^{2}}{2 C}
$$

where

$$
\begin{aligned}
& C=\left\{\lambda(1-\alpha) p_{1}-\lambda(1-\alpha)+1\right\}^{2}+\lambda^{2}(1-\alpha)^{2} p_{2}^{2}>0, \\
& A=2 \beta\left\{\lambda(1-\alpha) p_{1}-\lambda(1-\alpha)+1\right\}^{2}+\lambda^{2}(1-\alpha)^{2} p_{2}^{2}-\lambda(1-\beta)\left[\lambda(1-\alpha) p_{1}-\lambda(1-\alpha)+1\right],
\end{aligned}
$$

and

$$
B=-\lambda(1-\beta)\left[\lambda(1-\alpha) p_{1}-\lambda(1-\alpha)+1\right] .
$$

We note that $R e \psi\left(i u_{2}, v_{1}\right) \leq 0$ if and only if $A \leq 0$ and $B \leq 0$. From $A \leq 0$, we obtain $\beta \leq \beta_{A}$ where

$$
\beta_{A}=\frac{\lambda^{2}(1-\alpha)^{2} p_{2}^{2}+\lambda\left[\lambda(1-\alpha) p_{1}-\lambda(1-\alpha)+1\right]}{2\left[\left\{\lambda(1-\alpha) p_{1}-\lambda(1-\alpha)+1\right\}^{2}+\lambda^{2}(1-\alpha)^{2} p_{2}^{2}\right]+\left\{\lambda(1-\alpha) p_{1}-\lambda(1-\alpha)+1\right\}} \geq 0 .
$$

Also, from $B \leq 0$, we have $\beta_{A}<1$ and the condition (iii) is satisfied to give $R e p(z)>0$ for $z \in E$ which implies that $f \in K_{n}(\beta, \alpha)$.

If we put $n=\left(\frac{1}{\lambda}-1\right)$ in (3.1) we have the following:

THEOREM 3.2. Let $F \in K_{n}$ and let

$$
f(z)=(n+1) z-n \int_{0}^{z} \xi^{n-1} F(\xi) d \xi .
$$

Then $f \in K_{n+1}$.

PROOF. Let $g(z)=(n+1) z^{-n} \int_{0}^{z} \xi^{n-1} G(\xi) d \xi$,

where $G \in R_{n}$. Then from [2] $g \in R_{n+1}$. From (3.10) and (3.11) we have

$$
D^{n} F(z)=\frac{n}{n+1} D^{n} f(z)+\frac{1}{n+1} z\left(D^{n} f(z)\right)^{\prime}
$$

and

$$
D^{n} G(z)=\frac{n}{n+1} D^{n} g(z)+\frac{1}{n+1} z\left(D^{n} g(z)\right) .
$$

From (3.12) and the identity

$$
z\left(D^{n} f(z)\right)^{\prime}=(n+1) D^{n+1} f(z)-n D^{n} f(z),
$$

we have

$$
D^{n} F(z)=D^{n+1} f(z) .
$$

Since $F \in K_{n}$, it follows that, for $z \in E$,

$$
\operatorname{Re}\left(\frac{z\left(D^{n} F(z)\right)^{\dagger}}{D^{n} G(z)}\right)>0, \quad G \in R_{n}
$$


and thus, using (3.14), we conclude that, for $z \in E$

$$
\operatorname{Re}\left[\frac{z\left(D^{n+1} f(z)\right)^{\prime}}{D^{n+1} g(z)}\right]>0 \text { for } g \in R_{n+1} \text {. }
$$

THEOREM 3.3. Let $f \in K_{n}, n \geq 0$ and $\phi \in C$. Then $\phi^{*} f \in K_{n}$.

PROOF. First we prove that, if $g \in R_{n}$, then $\left(\phi^{*} g\right) \in R_{n}$. It is sufficient to show that $D^{n}\left(\phi^{*} g\right) \in S^{*}$.

Now

$$
D^{n}\left(\phi^{*} g\right)(z)=\frac{z}{(1-z)^{n+1}} *\left(g^{*} \phi\right)(z)=\phi(z) * \frac{z}{(1-z)^{n+1}} * g(z)=\left(\phi^{*} D^{n} g\right)(z) .
$$

Since $g \in R_{n}$ and $\phi \in C$, it follows that $\phi * g \in R_{n}$, see [2].

Next, we prove that $\left(\phi^{*} f\right) \in K_{n}$.

$$
\frac{z\left[D^{n}\left(\phi^{*} f\right)(z)\right]^{\prime}}{D^{n}\left(\phi^{*} g\right)(z)}=\frac{z\left[\phi(z)^{*} D^{n} f(z)\right]^{\prime}}{\phi(z)^{*} D^{n} g(z)}=\frac{\phi(z)^{*} z \frac{\left(D^{n} f(z)\right)^{\prime}}{D^{n} g(z)} \cdot D^{n} g(z)}{\phi(z)^{*} D^{n} g(z)}
$$

Applying Lemma 1.3 with $F(z)=\frac{z\left(D^{n} f(z)\right)^{\prime}}{D^{n} g(z)}, D^{n} g(z) \in S^{*}$ and $R e R(z)>0$, we obtain

$$
\operatorname{Re} \frac{z\left[D^{n}\left(\phi^{*} f\right)(z)\right]^{\natural}}{D^{n}\left(\phi^{*} f\right)(z)}>0 \text { for } z \in E
$$

This proves Theorem 3.3.

REMARK 3.1. Theorem 3.3 is an analogue of the Polya-Schoenberg conjecture [6] for the family $K_{n}$. Many results on $K_{n}$ can be deduced as applications.

We give the following:

THEOREM 3.4. Let $f \in K_{n}, n \geq 0$ and be defined by (3.1). Then $F \in K_{n}, n \geq 0$ for $|z|<r_{0}$, where $r_{0}$ is given by

$$
r_{0}=\frac{1}{\left(2 \lambda+\sqrt{\left.4 \lambda^{2}-2 \lambda+1\right)} .\right.}
$$

The function $f_{0}$, defined by (2.10), shows that this result is sharp.

PROOF. Let $\phi_{\lambda}(z)=\sum_{k=1}^{\infty}[\lambda(k-1)+1] z^{k}, 0<\lambda<1$.

Then $\lambda_{\phi} \in C$ for $|z|<r_{0}$ where $r_{0}$ is given by (3.15). Also $F(z)=\left(\phi_{\lambda}{ }^{*} f(z)\right)$ and so using Theorem 3.3, we see that $F \in K_{n}, n \geq 0$ for $|z|<r_{0}$.

REMARK 3.2. We note that Theorem 3.3 shows that the family $K_{n}$ is invariant under the following integral operators

$$
\begin{aligned}
I_{1}(f) & =\int_{0}^{z} \frac{f(\xi)}{\xi} d \xi=\left(f^{*} \phi_{1}\right)(z) \\
I_{2}(f) & =\frac{2}{2} \int_{0}^{z} \frac{f(\xi)}{\xi} d \xi=\left(f^{*} \phi_{2}\right)(z), \quad \text { (Libera's operator) } \\
I_{3}(f) & =\int_{0}^{z} \frac{f(\xi)-f(x \xi)}{\xi-x \xi} d \xi,|x|<1, x \neq 1 \\
& =\left(f^{*} \phi_{3}\right)(z)
\end{aligned}
$$


and

$$
\begin{aligned}
I_{4}(f) & =\frac{1+c}{c} \int_{0}^{z} \xi^{c-1} f(\xi) d \xi, & \operatorname{Re}(c)>0 \\
& =\left(f^{*} \phi_{4}\right)(z) &
\end{aligned}
$$

where $\phi_{i} \in C, i=1,2,3,4$

and

$$
\begin{aligned}
& \phi_{1}(z)=\sum_{n=1}^{\infty} \frac{1}{n} z^{n}=-\log (1-z), \\
& \phi_{2}(z)=\sum_{n=1}^{\infty} \frac{2}{n+1} z^{n}=\frac{-2[z+\log (1-z)]}{z}, \\
& \phi_{3}(z)=\sum_{n=1}^{\infty} \frac{1-x^{n}}{n(1-x)} z^{n}=\frac{1}{1-x} \log \frac{1-x z}{1-z},|x|=1, x \neq 1,
\end{aligned}
$$

and

$$
\phi_{4}(z)=\sum_{n=1}^{\infty} \frac{1+c}{n+c} z^{n}, \quad \operatorname{Re}(c)>0
$$

ACKNOWLEDGEMENT. The author would like to thank the referee whose comments influenced the final version of the paper.

\section{REFERENCES}

1. KAPLAN, W., Close-to-convex schlicht functions, Mich. Math. J. 1 (1952), 169-185.

2. AHUJA, $O$., Integral operators of certain univalent functions, Int. J. Math. and Math. Sci. 8 (1985), 653-662.

3. NOOR, K.I. \& THOMAS, D.K., On quasi-convex univalent functions, Int. J. Math. and Math Sci. 3 (1980), 225-266.

4. JACK, I., Functions starlike and convex of order $\alpha$, J. Lond. Math. Soc. 3 (1971), 469-474.

5. MILLER, S., Differential inequalities and Caratheordory functions, Bull. Amer. Math. Soc. 81 (1975), 79-81.

6. RUSCHEWEYH, ST. \& SHIEL-SMALL, T., Hadamard products of schlicht functions and the Polya-Schoenberg conjecture, Comment. Math. Helv. 48 (1973), 119-135.

7. GOODMAN, A.W., Univalent Functions, Mariner Publishing Company Inc., 1983.

8. LIBERA, R., Some radius of convexity problems, Duke Math. J. (1964), 143-158.

9. BERNARDI, S., Convex and starlike univalent functions, Trans. Amer. Math. Soc. 135 (1969), 429-446. 


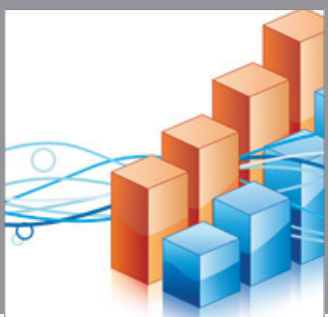

Advances in

Operations Research

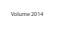

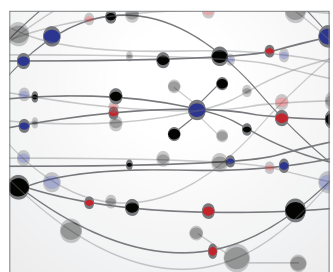

\section{The Scientific} World Journal
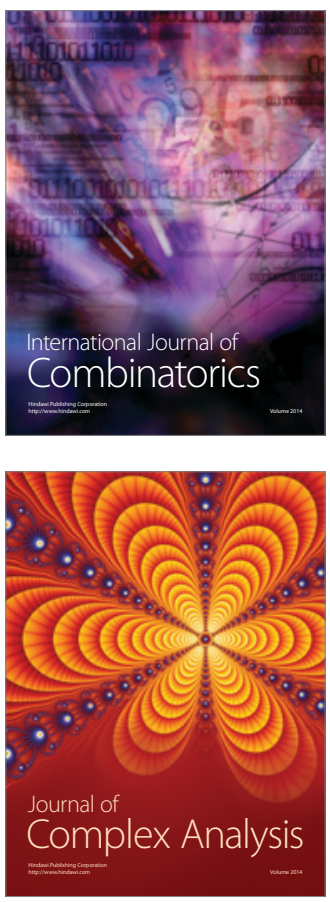

International Journal of

Mathematics and

Mathematical

Sciences
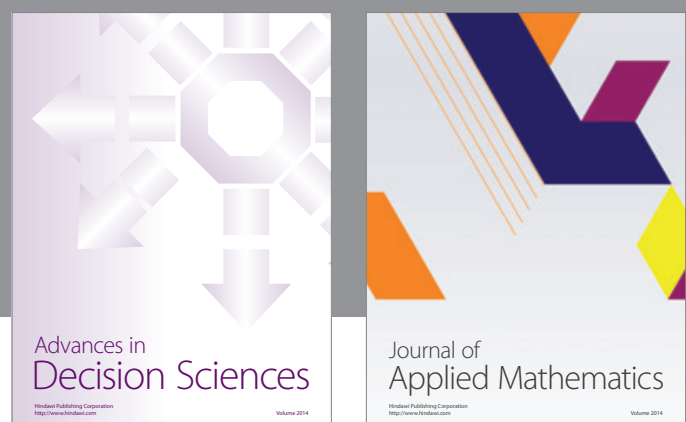

Journal of

Applied Mathematics
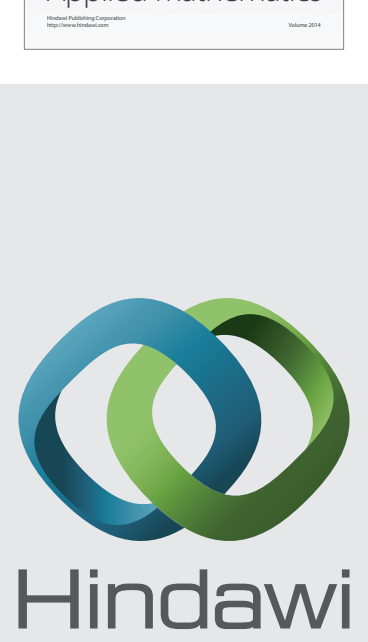

Submit your manuscripts at http://www.hindawi.com
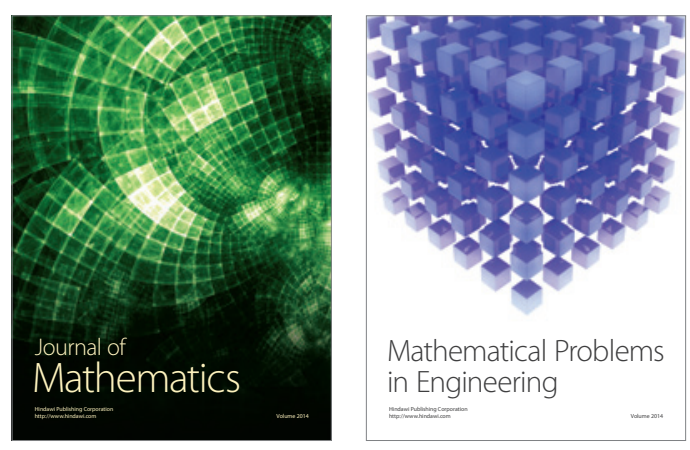

Mathematical Problems in Engineering
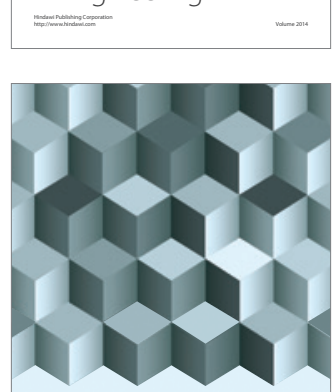

Journal of

Function Spaces
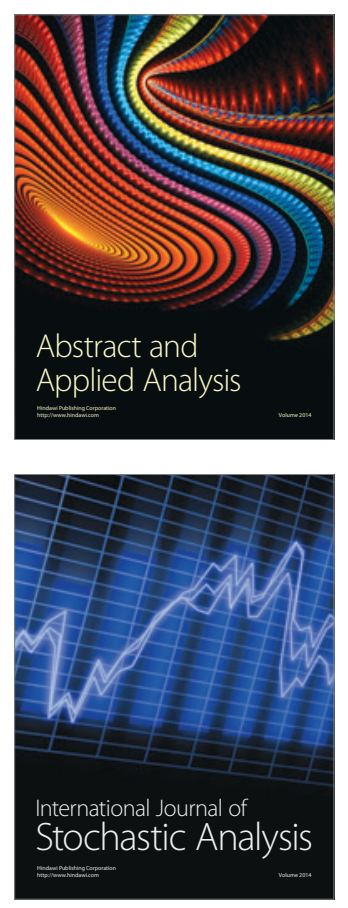

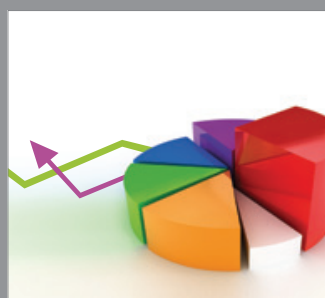

ournal of

Probability and Statistics

Promensencen
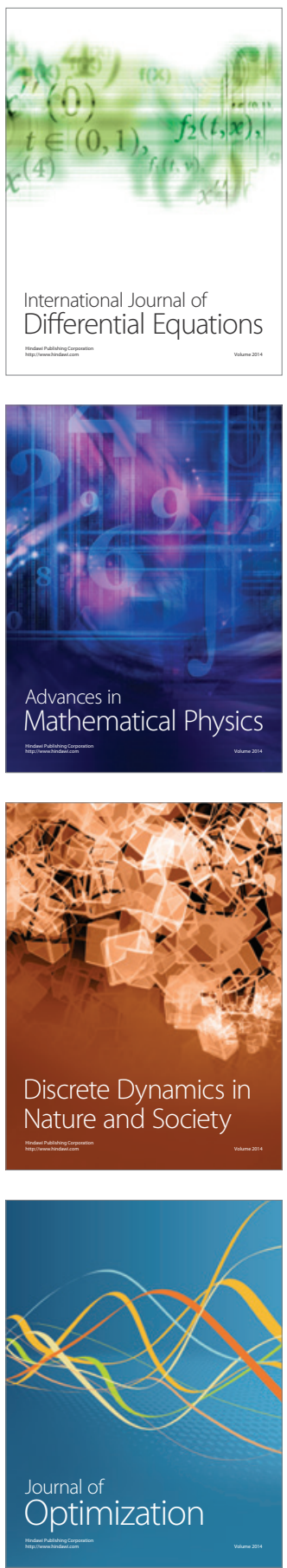